\title{
Virtual Prediction of Thymoquinone and $\beta$-Glucan Interaction as Estrogen Receptor-Alpha (ER- $\alpha$ receptor) May Reducing on The Breast Cancer Signaling Pathway
}

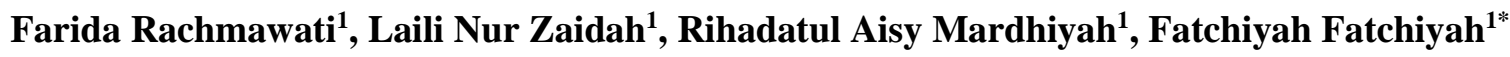 \\ ${ }^{1}$ Department of Biology, Faculty of Mathematical and Natuaral Sciences, Universitas Brawijaya \\ Jalan Veteran Malang East Java Indonesia 65145
}

Submission: August 2019; Revised: September 2019: Accepted: October 2019

* Corresponding author: Fatchiyah Fatchiyah; e-mail: fatchiyah@ub.ac.id; tel.: +62-341-575841

\begin{abstract}
Many cases of chemical drug treatment in breast cancer patients result in a negative impact on drug resistance. Therefore the use of natural compounds such as $\beta$-glucan on ajwa dates and thymoquinone on black cumin was expected to stop the process of cancer cell proliferation and to consume in long-term safety. This study aimed to predict the effect of thymoquinone and $\beta$-glucan in reducing breast cancer cascade with molecular docking. This research was carried out in silico. The ligand and protein preparations were done using Discovery Studio 2016. We used Hex 8.0.0 for docking. Visualization was established using Discovery Studio 2016 as well. The results showed that thymoquinone and $\beta$-glucan can undergo conformational changes in ligand binding domain (LBD) of ER- $\alpha$ receptor. The interaction between ER- $\alpha$ receptor with $\beta$-estradiol (inhibited by thymoquinone- $\beta$-glucan) was suggested to be the best solution in downregulating breast cancer signaling pathway. This interaction showed more stable conformation with the smallest binding energy $(-332,84 \mathrm{kcal} / \mathrm{mol})$. The thymoquinone- $\beta$-glucan complex could block the His476 and Met 438 .
\end{abstract}

Keywords: thymoquinone; $\beta$-glucan; $\beta$-estradiol; ER- $\alpha$ receptor; breast cancer

\section{INTRODUCTION}

The American Cancer Society has shown that around 1.3 billion people in the world are diagnosed with breast cancer each year with 465,000 reported to have died. The cases of women breast cancer increased to around 92,600 in Africa in 2008, with a mortality rate reaching $54 \%(50,000)$ [1,2]. The Ministry of Health of the Republic of Indonesia (2019) supported by the data from Basic Health Research (Riskesdas) stated that the prevalence of cancer in Indonesia reaches 136.2/100,000 population, being the number 8 in Southeast Asia. The most common type of cancer occurs in Indonesia, especially in women, is breast cancer, which is equal to 42.1 per 100,000 population with an average mortality rate reaching $17 / 100,000$ population [3]. This cancer attacks woman aged 30 years and over associated with menopausal events. One way to detect breast cancer is through the presence of estrogen receptors. About $75 \%$ of breast cancer is due to the response of estrogen hormone accumulation, mainly ER- $\alpha$ receptor. The number of ER- $\alpha$ receptors in the percentage of $10-20 \%$ is said to be normal. It will increase when the tumor begins to develop in relation to atypia conditions and reduction in ductal carcinoma in situ (DCIS). The chemical-based treatment such as Tamoxifen provides drug resistance even causes metastasis of breast tumors, so there is a need for natural drugs without side effects [4]. One of the natural drugs that can be developed is the thymoquinone compound from black cumin. It can reduce the activity of estrogen receptor proliferation [5]. The $\beta$-glucan compounds in ajwa dates (Phoenix dactylifera $\mathrm{L}$.) also play a role in treating carcinoma in-vivo and as anticancer drugs [6], inhibiting proliferation of breast cancer cells and increasing cell death [7]. The $\beta$-glucan acts as an adjuvant in improving the immune response in chemotherapy patients [8]. We predicted that thymoquinone in black cumin, $\beta$-glucan in Ajwa dates, or a combination of both compounds are effective as natural remedies for breast cancer sufferers. Therefore, we investigated the effectiveness of thymoquinone and $\beta$-glucan in inhibiting alpha estrogen receptors using molecular docking in this study.

\section{METHODS}

\section{Ligands Preparation}

Thymoquinone (CID: 10281), $\beta$-glucan (CID: 439262), and $\beta$-estradiol (CID: 5757) ligands were downloaded from http://pubchem.ncbi.nlm.nih.gov/ as 3D-SDF format. The energy of ligands was minimized and the ligands were converted to PDB format by PyRx. 
Table 1. Types of bond occured between ligands and amino acid residues of ER- $\alpha$ receptor

\begin{tabular}{|c|c|c|c|c|c|c|c|}
\hline Interaction & $\begin{array}{c}\text { Point } \\
\text { Interaction }\end{array}$ & $\begin{array}{l}\text { Distance } \\
(\AA)\end{array}$ & $\begin{array}{c}\text { From } \\
\text { Chemistry }\end{array}$ & $\begin{array}{c}\text { To } \\
\text { Chemistry }\end{array}$ & Type & $\begin{array}{l}\text { Chemistry } \\
\text { Bond }\end{array}$ & $\begin{array}{l}\text { Energy } \\
\text { Binding }\end{array}$ \\
\hline \multirow{5}{*}{$\begin{array}{l}\text { ER- } \alpha \text { receptor } \\
+\beta \text {-Estradiol }\end{array}$} & $\begin{array}{l}\text { B:MET438: } \\
\text { SD-:LIG1 }\end{array}$ & 5,11 & Sulfur & Pi-Orbitals & Pi-Sulfur & Other & \multirow{5}{*}{-262.25} \\
\hline & $\begin{array}{l}\text { B:MET437-: } \\
\text { LIG1 }\end{array}$ & 4,97 & Alkyl & Alkyl & Alkyl & Hydrophobic & \\
\hline & $\begin{array}{l}\text { B:MET437-: } \\
\text { LIG1 }\end{array}$ & 5,38 & Alkyl & Alkyl & Alkyl & Hydrophobic & \\
\hline & $\begin{array}{l}\text { :LIG1- } \\
\text { B:MET437 }\end{array}$ & 4,62 & Alkyl & Alkyl & Alkyl & Hydrophobic & \\
\hline & $\begin{array}{l}\text { A:HIS476-: } \\
\text { LIG1:C }\end{array}$ & 5,01 & Pi-Orbitals & Alkyl & Pi-Alkyl & Hydrophobic & \\
\hline \multirow{9}{*}{$\begin{array}{l}\text { ER- } \alpha \text { receptor } \\
+ \text { thymoquinone }\end{array}$} & $\begin{array}{l}\text { A:ARG434: } \\
\text { CD-B:LIG1:O }\end{array}$ & 3,06 & H-Donor & H-Acceptor & $\begin{array}{c}\text { Carbon } \\
\text { Hydrogen Bond }\end{array}$ & Hydrophobic & \multirow{9}{*}{-196.09} \\
\hline & $\begin{array}{l}\text { B:HIS476- } \\
\text { B:LIG1 }\end{array}$ & 5,40 & Pi-Orbitals & Pi-Orbitals & Pi-Pi Stacked & Hydrophobic & \\
\hline & $\begin{array}{l}\text { B:LIG1:C- } \\
\text { B:LEU469 }\end{array}$ & 3,72 & Alkyl & Alkyl & Alkyl & Hydrophobic & \\
\hline & $\begin{array}{l}\text { B:LIG1:C- } \\
\text { B:LYS472 }\end{array}$ & 3,03 & Alkyl & Alkyl & Alkyl & Hydrophobic & \\
\hline & $\begin{array}{l}\text { B:LIG1:C- } \\
\text { A:MET437 }\end{array}$ & 4,91 & Alkyl & Alkyl & Alkyl & Hydrophobic & \\
\hline & $\begin{array}{l}\text { B:LIG1:C- } \\
\text { B:LEU469 }\end{array}$ & 3,67 & Alkyl & Alkyl & Alkyl & Hydrophobic & \\
\hline & $\begin{array}{l}\text { B:HIS476- } \\
\text { B:LIG1:C }\end{array}$ & 4,58 & Pi-Orbitals & Alkyl & Pi-Alkyl & Hydrophobic & \\
\hline & $\begin{array}{l}\text { B:LIG1- } \\
\text { A:MET437 }\end{array}$ & 4,66 & Pi-Orbitals & Alkyl & Pi-Alkyl & Hydrophobic & \\
\hline & $\begin{array}{l}\text { B:LIG1- } \\
\text { B:LYS472 }\end{array}$ & 5,03 & Pi-Orbitals & Alkyl & Pi-Alkyl & Hydrophobic & \\
\hline \multirow{5}{*}{$\begin{array}{l}\text { ER- } \alpha \text { receptor } \\
+ \text { thymoquinone } \\
+\beta \text {-estradiol }\end{array}$} & $\begin{array}{l}\text { B:MET438: } \\
\text { SD-:LIG1 }\end{array}$ & 5,06 & Sulfur & Pi-Orbitals & Sulfur & Other & \multirow{5}{*}{-254.05} \\
\hline & $\begin{array}{l}\text { B:MET437-: } \\
\text { LIG1 }\end{array}$ & 5,03 & Alkyl & Alkyl & Alkyl & Hydrophobic & \\
\hline & $\begin{array}{l}\text { B:MET437-: } \\
\text { LIG1 }\end{array}$ & 5,44 & Alkyl & Alkyl & Alkyl & Hydrophobic & \\
\hline & $\begin{array}{l}\text { :LIG1- } \\
\text { B:MET437 }\end{array}$ & 4,64 & Alkyl & Alkyl & Alkyl & Hydrophobic & \\
\hline & $\begin{array}{l}\text { A:HIS476-: } \\
\text { LIG1:C }\end{array}$ & 4,94 & Pi-Orbitals & Alkyl & Pi-Alkyl & Hydrophobic & \\
\hline \multirow{6}{*}{$\begin{array}{l}\text { ER- } \alpha \text { receptor } \\
+\beta \text {-glucan }\end{array}$} & $\begin{array}{l}\text { A:ARG434: } \\
\text { HH22-B:LIG1:O }\end{array}$ & 2,57 & H-Donor & H-Acceptor & $\begin{array}{c}\text { Conventional } \\
\text { Hydrogen Bond }\end{array}$ & $\begin{array}{l}\text { Hydrogen } \\
\text { Bond }\end{array}$ & \multirow{6}{*}{$-349,32$} \\
\hline & $\begin{array}{l}\text { B:LIG1:H- } \\
\text { A:ALA430:O }\end{array}$ & 3,07 & H-Donor & H-Acceptor & $\begin{array}{c}\text { Carbon } \\
\text { Hydrogen Bond }\end{array}$ & $\begin{array}{l}\text { Hydrogen } \\
\text { Bond }\end{array}$ & \\
\hline & $\begin{array}{l}\text { B:LIG1:H- } \\
\text { A:ASP426:O }\end{array}$ & 2,88 & H-Donor & H-Acceptor & $\begin{array}{c}\text { Carbon } \\
\text { Hydrogen Bond }\end{array}$ & $\begin{array}{l}\text { Hydrogen } \\
\text { Bond }\end{array}$ & \\
\hline & $\begin{array}{l}\text { B:LIG1:H- } \\
\text { A:ASP426:O }\end{array}$ & 2,86 & H-Donor & H-Acceptor & $\begin{array}{c}\text { Carbon } \\
\text { Hydrogen Bond }\end{array}$ & $\begin{array}{l}\text { Hydrogen } \\
\text { Bond }\end{array}$ & \\
\hline & $\begin{array}{l}\text { B:LIG1:H- } \\
\text { B:LEU469:O }\end{array}$ & 2,28 & H-Donor & H-Acceptor & $\begin{array}{c}\text { Carbon } \\
\text { Hydrogen Bond }\end{array}$ & $\begin{array}{l}\text { Hydrogen } \\
\text { Bond }\end{array}$ & \\
\hline & $\begin{array}{l}\text { B:LIG1:H- } \\
\text { :ASP473:OD1 }\end{array}$ & 2,52 & H-Donor & H-Acceptor & $\begin{array}{c}\text { Carbon } \\
\text { Hydrogen Bond }\end{array}$ & $\begin{array}{l}\text { Hydrogen } \\
\text { Bond }\end{array}$ & \\
\hline
\end{tabular}


Table 1. Types of bond occured between ligands and amino acid residues of ER- $\alpha$ receptor (Continue)

\begin{tabular}{|c|c|c|c|c|c|c|c|}
\hline Interaction & $\begin{array}{c}\text { Point } \\
\text { Interaction }\end{array}$ & $\begin{array}{l}\text { Distance } \\
(\AA)\end{array}$ & $\begin{array}{c}\text { From } \\
\text { Chemistry }\end{array}$ & $\begin{array}{c}\text { To } \\
\text { Chemistry }\end{array}$ & Type & $\begin{array}{c}\text { Chemistry } \\
\text { Bond }\end{array}$ & $\begin{array}{l}\text { Energy } \\
\text { Binding }\end{array}$ \\
\hline \multirow{7}{*}{$\begin{array}{l}\text { ER- } \alpha \text { receptor } \\
+\beta \text {-glucan }+ \\
\beta \text {-estradiol }\end{array}$} & $\begin{array}{l}\text { A:LEU462: } \\
\text { CD1-:LIG1 }\end{array}$ & 3,89 & $\mathrm{C}-\mathrm{H}$ & Pi-Orbitals & Pi-Sigma & Hydrophobic & \multirow{7}{*}{$-260,33$} \\
\hline & $\begin{array}{l}\text { A:PHE461:C,O } \\
\text {;LEU462:N-LIG1 }\end{array}$ & 4,44 & Amide & Pi-Orbitals & $\begin{array}{l}\text { Amide-Pi } \\
\text { Stacked }\end{array}$ & Hydrophobic & \\
\hline & $\begin{array}{l}\text { A:LEU462-: } \\
\text { LIG1 }\end{array}$ & 5,26 & Alkyl & Alkyl & Alkyl & Hydrophobic & \\
\hline & $\begin{array}{l}\text { A:LEU462-: } \\
\text { LIG1 }\end{array}$ & 5,25 & Alkyl & Alkyl & Alkyl & Hydrophobic & \\
\hline & $\begin{array}{l}\text { B:ALA430-: } \\
\text { LIG1 }\end{array}$ & 4,75 & Alkyl & Alkyl & Alkyl & Hydrophobic & \\
\hline & $\begin{array}{l}\text { B:ALA430-: } \\
\text { LIG1 }\end{array}$ & 5,26 & Alkyl & Alkyl & Alkyl & Hydrophobic & \\
\hline & $\begin{array}{l}\text { :LIG1-B: } \\
\text { LEU429 }\end{array}$ & 5,27 & Alkyl & Alkyl & Alkyl & Hydrophobic & \\
\hline \multirow{7}{*}{$\begin{array}{l}\text { ER- } \alpha \text { receptor } \\
+ \\
\text { thymoquinone } \\
+\beta \text {-glucan }\end{array}$} & $\begin{array}{l}\text { B:LIG1:H- } \\
\text { B:ASP480:OD1 }\end{array}$ & 2,50 & H-Donor & H-Acceptor & $\begin{array}{c}\text { Conventional } \\
\text { Hydrogen Bond }\end{array}$ & $\begin{array}{l}\text { Hydrogen } \\
\text { Bond }\end{array}$ & \multirow{7}{*}{$-332,84$} \\
\hline & $\begin{array}{l}\text { B:IS476:CD2- } \\
\text { B:LIG1:O }\end{array}$ & 2,49 & H-Donor & H-Acceptor & $\begin{array}{c}\text { Carbon } \\
\text { Hydrogen Bond }\end{array}$ & $\begin{array}{l}\text { Hydrogen } \\
\text { Bond }\end{array}$ & \\
\hline & $\begin{array}{l}\text { B:LIG1:H- } \\
\text { B:ASP480:OD2 }\end{array}$ & 2,14 & H-Donor & H-Acceptor & $\begin{array}{c}\text { Carbon } \\
\text { Hydrogen Bond }\end{array}$ & $\begin{array}{l}\text { Hydrogen } \\
\text { Bond }\end{array}$ & \\
\hline & $\begin{array}{l}\text { B:LIG1:H- } \\
\text { B:ASP473:OD1 }\end{array}$ & 2,55 & H-Donor & H-Acceptor & $\begin{array}{c}\text { Carbon } \\
\text { Hydrogen Bond }\end{array}$ & $\begin{array}{l}\text { Hydrogen } \\
\text { Bond }\end{array}$ & \\
\hline & $\begin{array}{l}\text { B:LIG1:H- } \\
\text { A:GLU502:OE2 }\end{array}$ & 2,60 & H-Donor & H-Acceptor & $\begin{array}{c}\text { Carbon } \\
\text { Hydrogen Bond }\end{array}$ & $\begin{array}{l}\text { Hydrogen } \\
\text { Bond }\end{array}$ & \\
\hline & $\begin{array}{l}\text { B:LIG1:C- } \\
\text { A:MET438 }\end{array}$ & 4,88 & Alkyl & Alkyl & Alkyl & Hydrophobic & \\
\hline & $\begin{array}{l}\text { B:LIG1:C- } \\
\text { A:MET438 }\end{array}$ & 4,39 & Alkyl & Alkyl & Alkyl & Hydrophobic & \\
\hline \multirow{5}{*}{$\begin{array}{l}\text { ER- } \alpha \text { receptor } \\
+(\text { thymoquinone } \\
+\beta \text {-glucan }) \\
+\beta \text {-estradiol }\end{array}$} & $\begin{array}{l}\text { A:ASP426: } \\
\text { OD1-:LIG1 }\end{array}$ & 4,11 & Negative & Pi-Orbitals & Pi-Anion & Electrostatic & \multirow{5}{*}{$-276,84$} \\
\hline & $\begin{array}{l}\text { B:LEU462: } \\
\text { CD1-:LIG1 }\end{array}$ & 3,36 & $\mathrm{C}-\mathrm{H}$ & Pi-Orbitals & Pi-Sigma & Hydrophobic & \\
\hline & $\begin{array}{l}\text { A:LEU429-: } \\
\text { LIG1 }\end{array}$ & 5,37 & Alkyl & Alkyl & Alkyl & Hydrophobic & \\
\hline & $\begin{array}{l}\text { A:ALA430-: } \\
\text { LIG1 }\end{array}$ & 4,26 & Alkyl & Alkyl & Alkyl & Hydrophobic & \\
\hline & $\begin{array}{l}\text { :LIG1-A: } \\
\text { LEU429 }\end{array}$ & 4,57 & Alkyl & Alkyl & Alkyl & Hydrophobic & \\
\hline
\end{tabular}

\section{Human Estrogen Receptor-Alpha (ER-a Receptor) Protein Preparation}

Human ER- $\alpha$ receptor protein was obtained from http://www.rcsb.org/pdb/ with PDB ID:1A52. The protein was prepared by Discovery Studio 2016 client 3.5 to remove ligands and water molecules.

\section{Receptor-Ligand Docking and Visualization}

Docking was done by Hex 8.0.0 to predict the possible interaction and energy binding of ER- $\alpha$ receptor with Thymoquinone, $\beta$-glucan, and $\beta$ estradiol. We established several protein-ligand interactions. They are ER- $\alpha$ receptor $+\beta$-estradiol, ER- $\alpha$ receptor + thymoquinone, ER- $\alpha$ receptor + thymoquinone $+\beta$-estradiol, ER- $\alpha$ receptor $+\beta$ glucan, ER- $\alpha$ receptor $+\beta$-glucan $+\beta$-estradiol, ER$\alpha$ receptor + thymoquinone $+\beta$-glucan, and ER- $\alpha$ receptor + (thymoquinone $+\beta$-glucan $)+\beta$-estradiol . The output of docking was visualized using Discovery Studio 2016 client 3.5 to identify the interactions.

\section{RESULTS AND DISCUSSIONS}

The binding of ER- $\alpha$ receptor and $\beta$-estradiol established a Pi-Sulfur and 4 hydrophobic interactions with the total energy of $-262,25 \mathrm{kcal} / \mathrm{mol}$ (Table 1). Those interactions involved three amino 
acid residues of ER- $\alpha$ receptor, such as His476 from the A subunit, Met437 and Met438 from the B subunit. The interaction of ER- $\alpha$ receptor and thymoquinone occurred in ligand binding domain with the binding energy of $-196,09 \mathrm{kcal} / \mathrm{mol}$. The
ER- $\alpha$ receptor and thymoquinone complex showed that thymoquinone could block the His 476 of ER- $\alpha$ receptor with the presence of hydrophobic bond. The $\beta$-estradiol inhibited by $\beta$-glucan could block the active site on Met437 and Met438 of ER- $\alpha$ receptor.

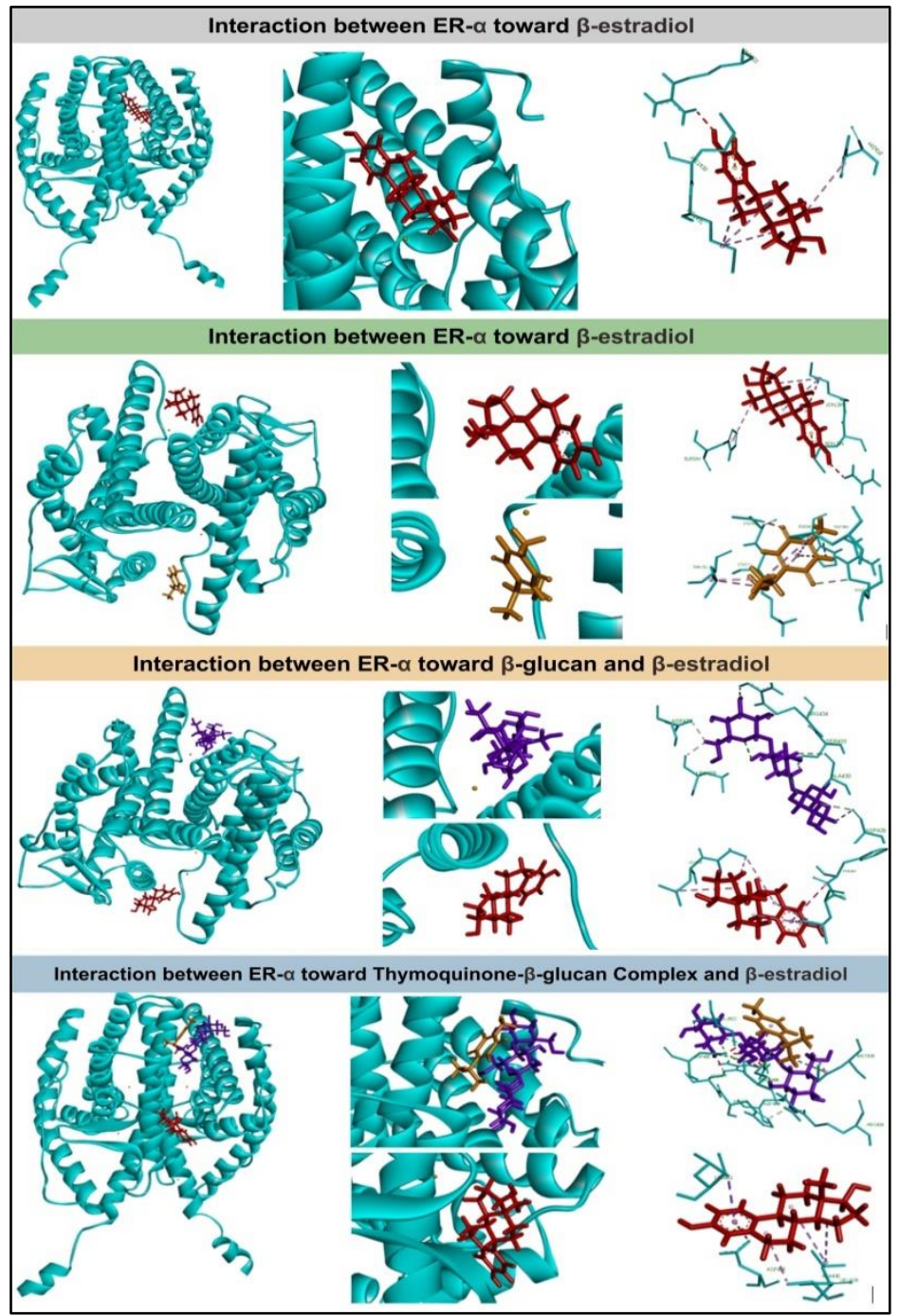

Figure 1. Illustration of ER- $\alpha$ receptor, $\beta$-estradiol, and ligands interaction: Thymoquinone (orange color) and $\beta$-glucan (purple color)

The $\beta$-glucan bound to ER- $\alpha$ receptor on ligand binding domain with five hydrogen bonds and had the smallest binding energy of $-349,32 \mathrm{kcal} / \mathrm{mol}$. Unfortunately, the $\beta$-glucan could not block the His 476, Met437 and Met438 of ER- $\alpha$ receptor. The thymoquinone- $\beta$-glucan complex bound to ER- $\alpha$ receptor by five hydrogen bonds and two hydrophobic interactions. This interaction had the binding energy of $-332,84 \mathrm{kcal} / \mathrm{mol}$. The $\beta$-estradiol interacted with ER- $\alpha$ receptor-Thymoquinone- $\beta$ glucan complex by an electrostatic and four hydrophobic interactions (Figure 1). The energy binding of the complex was -276.84 $\mathrm{kcal} / \mathrm{mol}$.Interaction between $\beta$-glucan and $\beta$ estradiol shown that the $\beta$-glucan could bind to the active site of ER- $\alpha$ receptor. It indicated that $\beta$ glucan would compete with $\beta$-estradiol. According to
Ostradini experiment [8], $\beta$-glucan compounds acted as adjuvants in enhancing the immune response in chemotherapy patients. The $\beta$-glucan might affect the estrogen secretion by ER- $\alpha$ receptor through interacting with the active side of ER- $\alpha$ receptor. Therefore, the over production of estrogen would be decreased.

The interaction of $\beta$-estradiol-thymoquinone complex with ER- $\alpha$ receptor in ligand binding domain showed that $55 \%$ of $\beta$-estradiolthymoquinone complex could be co-repressor binding in ER- $\alpha$ receptor and $\beta$-estradiol complex [9]. Thymoquinone was effective enough to inhibit the interaction between the native ligand ( $\beta$ estradiol) and ER- $\alpha$ receptor almost on all active sites (Met437, Met438). Therefore, thymoquinone could be predicted to inhibit breast cancer growth. But if it 
considered from the docking energy aspect, this second solution was less stable than docking solution via targeting ER- $\alpha$ receptor.

The ER- $\alpha$ receptor docking with $\beta$-glucan and $\beta$ Estradiol performed a difference in the binding site of ER- $\alpha$ receptor and $\beta$-estradiol, which is ER- $\alpha$ receptor origin ligand pair. It indicated that $\beta$-glucan did not bind to the active site of ER- $\alpha$ receptor- $\beta$ estradiol complex. The hydrogen bonds were identified on Asp473, Leu469, and Asp426 of ER- $\alpha$ receptor- $\beta$-glucan- $\beta$-estradiol complex. Furthermore, the unfavorable bump with hydrogen bond was also identified on Arg434. Hydrogen bond was very important for protein structure stability and it determined receptor specificity to ligand [10,11]. The binding energy was smallest than others ($349.32 \mathrm{kcal} / \mathrm{mol}$ ), more stable and stronger than Thymoquinone.

The combination of thymoquinone and $\beta$-glucan in the form of ligand complex could disturb the $\beta$ estradiol binding on ligand binding domain (LBD) of ER- $\alpha$ receptor. As a result, $\beta$-estradiol binding site to ER- $\alpha$ receptor turned into Asp426, Leu462, Leu429, Ala 430, and Leu429 residues which were different from standard. Thymoquinone- $\beta$-glucan complex made strong bond to ER- $\alpha$ receptor because it involved 5 hydrogen bonds with Asp480, His476, Asp473, and Glu502 from the B subunit. This interaction affected to the total energy used in the docking. The energy used by them $(-332.84$ $\mathrm{kcal} / \mathrm{mol}$ ) was lower than the energy used by interaction between ER- $\alpha$ receptor and $\beta$-estradiol in native condition $(-262.25 \mathrm{kcal} / \mathrm{mol})$.

\section{CONCLUSION}

The combination of thymoquinone and $\beta$-glucan might have potential as ER- $\alpha$ receptor inhibitor, which bind to ligand binding domain (LBD) of ER$\alpha$ receptor and disturb the interaction between ER- $\alpha$ receptor and $\beta$-estradiol as native ligand. Therefore, the combination of thymoquinone and $\beta$-glucan is considered as the best solution in downregulating breast cancer signaling pathway.

\section{ACKNOWLEDGEMENTS}

We send our regards to our research group especially to Hazna Noor Meidinna for helping us to finish this research.

\section{REFERENCES}

1. De Santis, C., M. Jiemin, B. Leah \& J. Ahmedin, Breast Cancer Statistics, 2013, Pp. 1-11.

2. GLOBOCAN, Cancer in Africa, http://globocan.iarc.fr, 2008, Accessed on March, 18th 2019.

3. Kementrian Kesehatan Republik Indonesia, Hari Kanker Sedunia, 2019, www.depkes.go.id. Accessed on March, 18th 2019.

4. Yared, Y.G \& A.B. Birhane, IJPSR, 2015, 6(8). Pp. 1100-1104.

5. Motaghed, M., American Journal of Life Sciences, 2015, 3(2), Pp. 7-14.

6. Khan,. M.A., T. Mousumi., F. Shangyi, \& F. Junjiang, Oncotarget Review, 2017, 8 (31). Pp. 51907-51919.

7. Jafaar Z.M.T., M.L Lacey., M.I Margarita., N.R Brandie., A. Numan \& M.K Carolyn, International of Oncology, 2014, 44(1), Pp. 1365-1375.

8. Ostadrahimi, A., E. Ali., A.J Mohammad., E.Z Jamal., M. Aliakbar \& F. Nazila, Adv Pharm Bull. 2014, 4(1), Pp. 471-477.

9. Chamkasem, A. \& T. Waraphan, Asian Pac J Cancer Prev, 2016, 16(6), Pp. 2161-2166.

10. Hoffman, R., E. J. Benz, L. E. Silberstein, H. E. Heslop, J. I. Weitz, J. Anastasi, M. E. Salama, \& S.A. Abutalib, Hematology: Basic Principles and Practice 7th Edition, 2018, Elsevier, Amsterdam.

11. Wermuth, C. G., D. Aldous, P. Raboisson, \& D. Rognan, the Practice of Medicinal Chemistry 4th Edition, 2015, Elsevier, Amsterdam. 